\title{
Tomographic reconstruction of circularly polarized high-harmonic fields: 3D attosecond metrology
}

2016 (c) The Authors, some rights reserved; exclusive licensee American Association for the Advancement of Science. Distributed under a Creative Commons Attribution NonCommercial License 4.0 (CC BY-NC). $10.1126 /$ sciadv. 1501333

\section{Cong Chen, ${ }^{1 *}$ Zhensheng Tao, ${ }^{1 *}$ Carlos Hernández-García, ${ }^{1,2}$ Piotr Matyba, ${ }^{1}$ Adra Carr, ${ }^{1}$ Ronny Knut, ${ }^{1}$ Ofer Kfir, ${ }^{3}$ Dimitry Zusin,, Christian Gentry, ${ }^{1}$ Patrik Grychtol, ${ }^{1}$ Oren Cohen, ${ }^{3}$ Luis Plaja, ${ }^{2}$ Andreas Becker, ${ }^{1}$ Agnieszka Jaron-Becker, ${ }^{1}$ Henry Kapteyn, ${ }^{1}$ Margaret Murnane ${ }^{1}$}

\begin{abstract}
Bright, circularly polarized, extreme ultraviolet (EUV) and soft x-ray high-harmonic beams can now be produced using counter-rotating circularly polarized driving laser fields. Although the resulting circularly polarized harmonics consist of relatively simple pairs of peaks in the spectral domain, in the time domain, the field is predicted to emerge as a complex series of rotating linearly polarized bursts, varying rapidly in amplitude, frequency, and polarization. We extend attosecond metrology techniques to circularly polarized light by simultaneously irradiating a copper surface with circularly polarized high-harmonic and linearly polarized infrared laser fields. The resulting temporal modulation of the photoelectron spectra carries essential phase information about the EUV field. Utilizing the polarization selectivity of the solid surface and by rotating the circularly polarized EUV field in space, we fully retrieve the amplitude and phase of the circularly polarized harmonics, allowing us to reconstruct one of the most complex coherent light fields produced to date.
\end{abstract}

\section{INTRODUCTION}

Tabletop extreme ultraviolet (EUV) and soft x-ray radiation from the high-harmonic generation (HHG) process are unique light sources for uncovering new fundamental understanding of dynamics in atoms $(1,2)$, molecules $(3)$, and materials $(4,5)$. However, until very recently, most experiments used linearly polarized harmonics that can easily be produced using single-color driving lasers. In this case, HHG emerges when an electron that is ionized by a laser field is driven back and recombines with its parent ion $(6,7)$. When implemented in a phase-matched geometry, the dipole emission from each atom interferes constructively to generate a bright HHG beam (8). However, until recently, it was not possible to generate bright, circularly polarized HHG for probing magnetic materials (9) or chiral molecules (10). This is because although atoms still undergo strong-field ionization in circularly polarized fields, the probability of an electron recombining with its parent ion to emit an HHG photon is greatly suppressed $(6,11,12)$.

Fortunately, high-brightness circularly polarized harmonics can now be produced in the EUV and soft $\mathrm{x}$-ray regions by driving HHG with bichromatic circularly polarized counter-rotating fields (13-21). In this scheme, a unique HHG spectrum is generated, consisting of pairs of peaks, each with opposite circular polarization. Theory predicts that for counter-rotating $\omega$ and $2 \omega$ laser fields, the circular harmonics are generated as a superposition of three bursts of linearly polarized EUV light per optical cycle in the time domain, where the polarization of each burst is rotated by $120^{\circ}$ from its predecessor $(17,19)$. Experimentally, the electron trajectories have been shown to predominantly move in a two-dimensional (2D) plane $(17,22)$. However, to date, no direct measurement of the temporal characteristics of circularly polarized HHG

${ }^{1}$ Department of Physics and JILA, University of Colorado, Boulder, Boulder, CO 803090440, USA. ${ }^{2}$ Grupo de Investigación en Aplicaciones del Láser y Fotónica, University of Salamanca, E-37008 Salamanca, Spain. ${ }^{3}$ Solid State Institute and Physics Department, Technion, Haifa 32000, Israel.

*Corresponding author. E-mail: cong.chen@colorado.edu (C.C.); zhensheng.tao@jila. colorado.edu (Z.T.) exists that could be used to inform and validate advanced theory and to harness the enormous potential of extreme nonlinear optics to generate arbitrary spectral, temporal, and polarization-shaped light fields spanning the EUV and soft $\mathrm{x}$-ray regions. This is due to the novelty of high-brightness circularly polarized HHG, their complex temporal structure, and the fact that conventional methods (23-28) for pulse characterization are not directly applicable. Moreover, it was recently proposed that, by using crossed beams of circularly polarized lasers or taking into account the orbital angular momentum of the initial state, either isolated circularly polarized single attosecond pulses $(29,30)$ or highly elliptical attosecond pulse trains $(31,32)$ could be produced. Therefore, the ability to directly characterize the temporal structure of circularly polarized harmonics would benefit a whole new area of polarization-shaped $\mathrm{x}$-ray attosecond science.

Here, by using laser-dressed angle-resolved photoemission from solids, we extend attosecond metrology techniques to circular polarization. By simultaneously irradiating a copper surface with circularly polarized HHG and linearly polarized infrared (IR) laser fields, the resulting modulation of photoelectron spectra caused by quantumpath interference carries essential phase information about the EUV field. Utilizing the strong polarization selectivity in photoemission from solid surfaces, as well as the ability to rotate the circularly polarized EUV field in space, we retrieve the full waveform of circularly polarized high-harmonic fields. This represents the first direct measurement of circularly polarized HHG in the time domain, enabling us to fully characterize one of the most complex coherent light fields to date, where the spectral, temporal, and polarization states are rapidly changing on attosecond time scales.

\section{RESULTS}

In our experiments shown in Fig. 1A, bright circularly polarized harmonics were generated by mixing a near-IR field $(\omega, 780 \mathrm{~nm})$ with its second harmonic $(2 \omega, 390 \mathrm{~nm})$ in a $1-\mathrm{cm}$-long waveguide filled with 


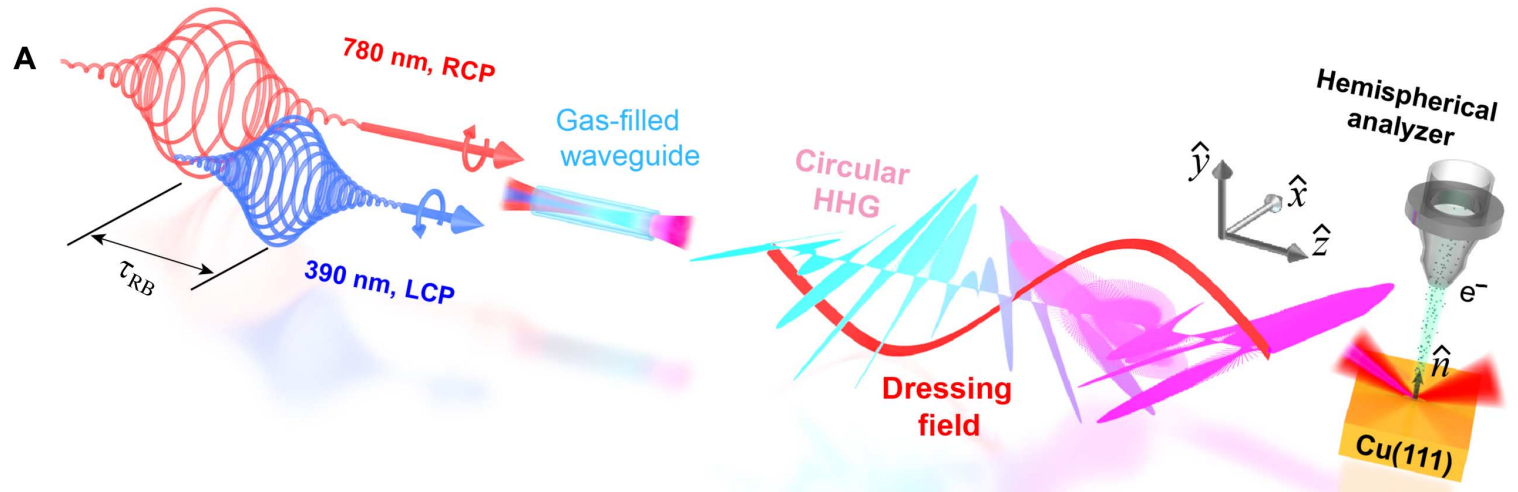

B

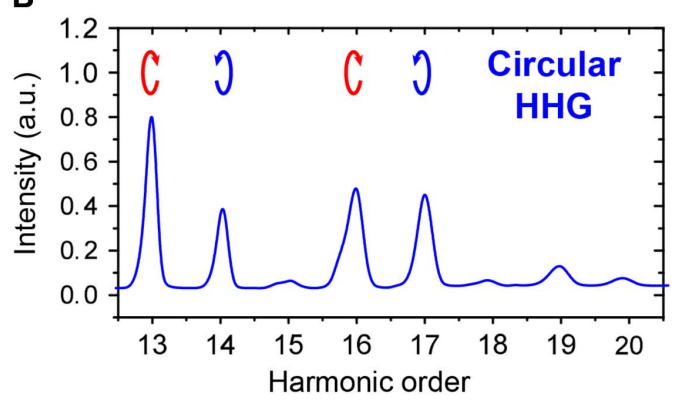

C

Photoelectron energy (eV)

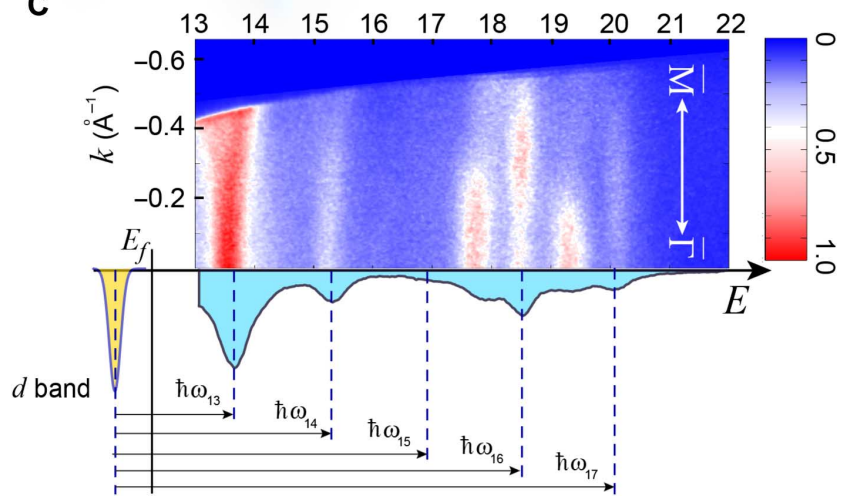

Fig. 1. Characterizing circularly polarized HHG fields using a laser-dressed angle-resolved photoelectron spectrometer. (A) Counter-rotating $\omega[780 \mathrm{~nm}$, right circularly polarized (RCP)] and $2 \omega[390 \mathrm{~nm}$, left circularly polarized (LCP)] beams from a Ti/Sapphire laser are focused into an Ar-filled hollow waveguide. The generated circular HHG and a time-delayed linearly polarized 780-nm dressing field are focused onto a clean $\mathrm{Cu}(111)$ surface. Here, a specific geometry is plotted for illustration, where the $\hat{y}$-pol component of circular HHG field is aligned with the normal direction of sample $(\hat{n})$. In experiments, the orientation of the circular HHG field can be rotated by adjusting the temporal delay between the $\omega$ and $2 \omega$ driving fields $\tau_{\mathrm{RB}}$. (B) Circularly polarized HHG spectrum recorded by an EUV spectrometer. The 13th and 16th harmonic orders are RCP, and the 14th and 17th orders are LCP. a.u., arbitrary unit. (C) Angle-resolved photoelectron spectrometer (ARPES) spectrum of $\mathrm{Cu}(111)$ excited by the circularly polarized HHG. The spectrum with momentum span $\overline{\Gamma \mathrm{M}}$ is recorded by a hemispherical electron analyzer. The analyzer work function is $4.2 \mathrm{eV}$ and correlates the photoelectron energy with photon energy. The direct photoemission pathways from $\mathrm{Cu} d$ band excited by the corresponding harmonic orders are labeled in the lower panel. $k$, momentum; $E_{f}$ Fermi level.

argon gas at a pressure of 30 torr (14). The helicities of the driving fields were adjusted to be right and left circularly polarized for $\omega$ and $2 \omega$, respectively, by using combinations of $\lambda / 2$ and $\lambda / 4$ waveplates in each $\mathrm{arm}$. The relative time delay between the two beams $\left(\tau_{\mathrm{RB}}\right)$ was controlled using a piezo stage with sub-femtosecond scanning precision (see the Supplementary Materials for a detailed experimental setup). The spectrum of circular harmonics consists of three pairs of harmonic orders [ $(13 \omega, 14 \omega),(16 \omega, 17 \omega)$, and $(19 \omega, 20 \omega)$ in Fig. 1B], each with right and left helicity, which is a consequence of photon energy and spin angular momentum conservation rules $(13,14)$. Consistent with experiment and theory $(13,14)$, the intensities of the $3 m$ th orders $(15 \omega$ and $18 \omega)$ are strongly suppressed because they do not conserve parity and spin angular momentum in the case of perfectly circularly polarized driving lasers. However, they are not completely suppressed because a slight ellipticity was introduced into the $2 \omega$ driving field to serve the characterization purposes.

Full characterization of the amplitude and phase of linearly polarized HHG fields has been demonstrated using many approaches based on an electron phase modulator (28), including atto-streaking (25),
RABBITT (resolution of attosecond beating by interference of twophoton transitions) $(24,33), \mathrm{CRAB}$ (complete reconstruction of attosecond bursts) (26), and PROOF (phase retrieval by omega oscillation filtering), which was successfully used to retrieve the phases associated with isolated attosecond pulses, necessarily with an ultrabroad spectrum (27). In these measurement schemes, the harmonic phases are imprinted as modulations on the photoelectron energy-momentum spectrum in the presence of a dressing (or streaking) IR laser field (28).

In our work, photoelectrons were ejected from an atomically clean $\mathrm{Cu}(111)$ surface by the circularly polarized harmonics while being simultaneously dressed by a linearly polarized IR field $\left(780 \mathrm{~nm}, \hbar \omega_{\mathrm{L}}=\right.$ $1.6 \mathrm{eV}$ ). The interference between different quantum pathways (Figs. $1 \mathrm{C}$ and $2 \mathrm{~B}$ ) induces a modulation of the photoelectron yield, which was recorded as a function of temporal delay $\left(\tau_{\mathrm{d}}\right)$ between the HHG and IR dressing fields using a hemispherical electron analyzer. Because of a marked difference in the photoemission cross section of the $\mathrm{Cu}(111)$ surface for s- and p-polarized EUV light (see the Supplementary Materials), photoelectrons ejected by an s-polarized field dominate the signal, that is, HHG polarization parallel to the sample surface. This 

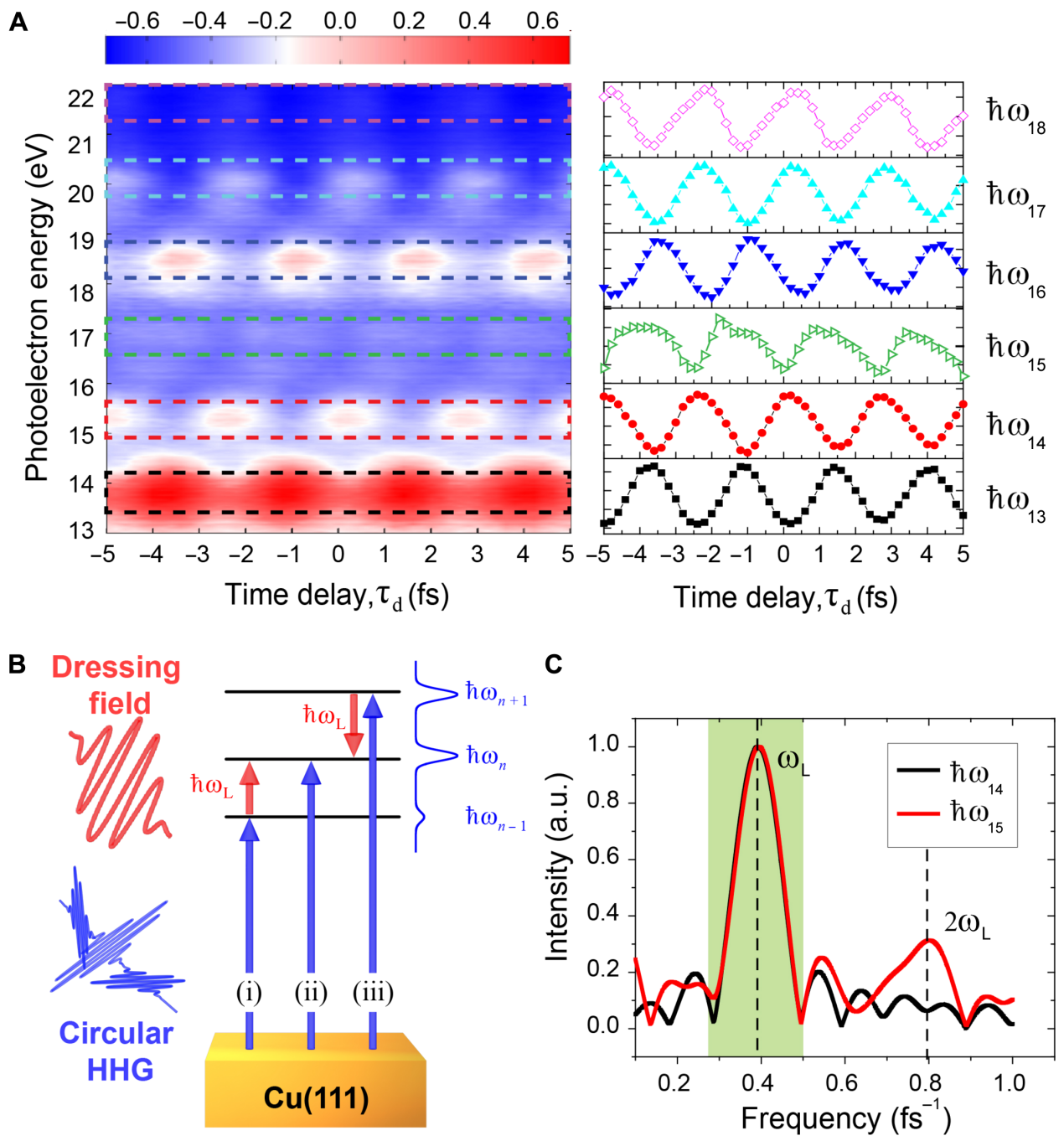

Fig. 2. Photoelectron interferogram as a function of time delay between the circular HHG and linear IR field. (A) Two-dimensional map of photoelectron yields as a function of photoelectron energy and pump-probe time delay. The selected lineouts on the right panel represent the photoelectronintensity modulation at the photoelectron energies corresponding to direct photoemission from the $\mathrm{Cu}(111) d$ band excited by labeled HHG orders. (B) Schematic of the quantum paths that give rise to photoelectron interferences. (C) Fourier analysis of the photoelectron yield oscillations at energies corresponding to direct photoemission by $14 \omega$ and $15 \omega$. The green area marks the frequency mask used to extract the phases of the $\omega_{\mathrm{L}}$ oscillations in (A).

eliminates not only ambiguities resulting from the entanglement of harmonic phases from two orthogonal polarizations $(\hat{x}$ and $\hat{y}$ shown in Fig. 1A) in the electron interferogram but also the use of EUV multilayer polarizers, which have very low efficiency and narrow bandwidth in this photon energy range. Moreover, a major advantage of our scheme is that a simple rotation of the HHG field (achieved by varying the delay between the $\omega$ and $2 \omega$ driving fields) allows us to record the perpendicular component of the HHG polarization.

The $\hat{x}$ polarization $(\hat{x}$-pol) of the circular harmonics was first aligned parallel to the sample surface. The temporal interferogram of photoelectrons, for $\tau_{\mathrm{d}}$ in the range of -5 to $5 \mathrm{fs}$, is plotted in Fig. 2A. These data were obtained by integrating the photoelectron spectra over the entire momentum range and subtracting the background photoelectron spectrum obtained well before time zero. To eliminate photoelectrons originating from different states in the material $(2,5)$, the angle-resolved spectra were filtered before integration, so that photoelectrons only from the $\mathrm{Cu} d$ band contributed to the interferogram, which have an almost constant photoemission cross section in the energy range of our experiments (34) (see the Supplementary Materials).

As can be seen in Fig. 2A, the photoelectron yields as a function of $\tau_{\mathrm{d}}$ oscillate with a frequency of $\omega_{\mathrm{L}}$ (periodicity of $2.6 \mathrm{fs}$ ), which can be understood through quantum-path interferences (Fig. 2B). Because the energy spacing between consecutive circularly polarized harmonics is $\hbar \omega_{\mathrm{L}}$ (Fig. 1B), there are three distinct quantum pathways for exciting electrons from a given initial state to the same final state: (i) absorbing an HHG and an IR photon ( $\left.\hbar \omega_{n-1}+\hbar \omega_{\mathrm{L}}\right)$, (ii) direct photoemission by a single HHG photon ( $\left(\omega_{n}\right)$, and (iii) absorbing an HHG photon and emitting an IR photon $\left(\hbar \omega_{n-1}-\hbar \omega_{\mathrm{L}}\right)$. The photoelectron yield oscillates with frequency $\omega_{\mathrm{L}}$ because of one-IR-photonassisted interference between quantum paths (i) and (ii), and (ii) and (iii). This cross section is much larger than that of two-IR-photonassisted interferences between (i) and (iii), which causes oscillations 
with frequency $2 \omega_{\mathrm{L}}$, as used in RABBITT characterizations (24) (see the Supplementary Materials). This conclusion is also supported by a Fourier analysis of the photoelectron yield oscillations (Fig. 2C), where the amplitude of the $2 \omega_{\mathrm{L}}$ component is very weak for the 15 th order and almost completely absent for the 14th order.

The phases of the harmonics are encoded in the $\omega_{\mathrm{L}}$ oscillations of the photoelectron yield interferogram. At the electron kinetic energy corresponding to direct photoemission by the $n$ th-order harmonics $\left(\hbar \omega_{n}\right)$, the oscillating spectral intensity as a function of $\tau_{\mathrm{d}}$ can be written in the general form

$$
S_{n}\left(\tau_{\mathrm{d}}\right)=\kappa\left(\omega_{n}\right) \sin \left[\omega_{\mathrm{L}} \tau_{\mathrm{d}}+\alpha\left(\omega_{n}\right)\right]
$$

where $\kappa\left(\omega_{n}\right)$ is the energy-dependent oscillation amplitude and $\alpha\left(\omega_{n}\right)$ is the phase spectrum of the interferogram, which is related to the phase spectrum of HHG $\phi\left(\omega_{n}\right)$ by

$\tan \left[\alpha\left(\omega_{n}\right)\right]=$

$$
\frac{\sqrt{I_{0}\left(\omega_{n+1}\right)} \sin \left[\phi\left(\omega_{n}\right)-\phi\left(\omega_{n+1}\right)\right]-\sqrt{I_{0}\left(\omega_{n-1}\right)} \sin \left[\phi\left(\omega_{n-1}\right)-\phi\left(\omega_{n}\right)\right]}{\sqrt{I_{0}\left(\omega_{n+1}\right)} \cos \left[\phi\left(\omega_{n}\right)-\phi\left(\omega_{n+1}\right)\right]-\sqrt{I_{0}\left(\omega_{n-1}\right)} \cos \left[\phi\left(\omega_{n-1}\right)-\phi\left(\omega_{n}\right)\right]}
$$

$I_{0}\left(\omega_{n}\right)$ is the power spectrum (see the Supplementary Materials for detailed derivations). Equation 1 bridges the interferogram phase $\left[\alpha\left(\omega_{n}\right)\right]$ and the phase differences of corresponding neighboring harmonics $\left[\phi\left(\omega_{n}\right)-\phi\left(\omega_{n+1}\right)\right]$ and $\left[\phi\left(\omega_{n-1}\right)-\phi\left(\omega_{n}\right)\right]$, allowing us to retrieve the harmonic phases from the interferogram in an iterative way. Here, the full interferogram phase spectra were fed into a genetic algorithm to retrieve the harmonic phases by minimizing the ranking function

$$
R=\sqrt{\sum_{\omega_{n}} I\left(\omega_{n}\right)\left[\alpha^{\text {calc }}\left(\omega_{n}\right)-\alpha^{\operatorname{expt}}\left(\omega_{n}\right)\right]^{2}}
$$

(see the Supplementary Materials), where $\alpha^{\text {calc }}\left(\omega_{n}\right)$ and $\alpha^{\operatorname{expt}}\left(\omega_{n}\right)$ are the interferogram phases obtained from the retrieval algorithm and the experiment, respectively.

Using the same procedure, we characterized the perpendicular polarization component ( $\hat{y}$-pol) by aligning it parallel to the sample surface. This was achieved by delaying the circularly polarized $\omega$ driving field by three-eighths of its wavelength $(292 \mathrm{~nm})$ relative to the counterrotating $2 \omega$ field. As a result, the orientation of the combined driving field (with threefold symmetry) (22), and hence the circularly polarized HHG field, is rotated by $90^{\circ}$. Although this rotation has a negligible influence on the HHG spectrum and flux, it has a significant impact on the photoelectron interferogram and the corresponding harmonic phases, allowing us to reconstruct the temporal profile of the $\hat{y}$-pol HHG field (see the Supplementary Materials). Our approach therefore extends the RABBITT (24) and PROOF (27) characterization approaches to be able to extract the $3 \mathrm{D}$ temporal-polarization structure of an arbitrary polarized attosecond pulse train. The retrieved phases of the $\hat{x}$ and $\hat{y}$ components of HHG fields are listed in Table 1.

With the knowledge of both the amplitude and phase, the temporal structure of the circularly polarized harmonics can be uniquely determined. The 3D electric field of the experimentally reconstructed circular HHG pulse train is plotted in Fig. 3. Three linearly polarized bursts of the EUV fields with different orientations can be clearly distinguished in each IR optical cycle (2.6 fs), in agreement with the pulse train structure generated by our numerical simulations (35) (see the Supplementary Materials for a comparison of the experimental and theoretical $E_{x}$ and $E_{y}$ components). A typical duration of the EUV bursts is $\sim 600$ as. As shown in Fig. $4 \mathrm{~A}$, the field orientation of the EUV bursts rotates approximately $120^{\circ}$ from one burst to the next, which is a consequence of the threefold symmetry of the combined driving field, directly validating previous theory (17).

From Eq. 1, we note that contributions from quantum pathways involving the suppressed $3 m$ th-order harmonics are essential for retrieving the harmonic phases because they allow us to bridge the neighboring pairs of strong harmonics that are separated by $2 \omega_{\mathrm{L}}$. The appearance of $3 m$ th-order harmonics is a direct consequence of a slight ellipticity in the driving laser fields, which was intentionally introduced into the $2 \omega$ driving beam. By comparing the measured normalized spectral intensity of the $3 m$ th-order harmonics $\left(\frac{2 I_{3 m}}{I_{3 m-1}+I_{3 m+1}}\right)$ with our numerical simulation, we determine the ellipticity of the $2 \omega$ field to be $0.92 \pm 0.04$ (see the Supplementary Materials). Previously, the ellipticity of circular HHG from Ne was characterized using x-ray magnetic circular dichroism (14). Using our novel approach to access the harmonic amplitudes, phases, and polarization direction, we can analyze the chiral properties of HHG in any spectral region, even when the photon energy does not overlap a characteristic absorption edge of a magnetic material.

\section{DISCUSSION}

The ellipticity $\varepsilon_{\mathrm{X}}$ of the circular harmonics is plotted in Fig. $4 \mathrm{C}$ and is also directly compared with our numerical simulations. We find that the $3 m+1$ th and $3 m-1$ th orders are mostly circularly polarized, whereas the $3 m$ th (that is, 15th) orders are strongly elliptical, consistent with previous studies and theory $(13,14)$. A reduction in the degree of HHG circularity can be observed in the cutoff region for $3 m+1$ th and $3 m-1$ th orders, which is also quantitatively confirmed by our numerical simulations. We note that this behavior is not predicted by perturbative models $(13,36,37)$. For linear HHG, it is known that the high-energy harmonics in the cutoff region are generated in a narrow temporal

Table 1. Phases of circular harmonics along $\hat{x}$ - and $\hat{y}$-pol (see Fig. 1A), obtained using a phase retrieval algorithm. The uncertainty is determined as the SD of phase values retrieved from multiple trials (see the Supplementary Materials).

\begin{tabular}{|c|c|c|c|c|c|c|}
\hline & \multicolumn{5}{|c|}{ Phases of the harmonics (rad) } \\
\hline Polarization & 13 th & 14 th & 15 th & 16 th & 17 th \\
\hline$\hat{x}$ & 0.0 & $0.57 \pm 0.29$ & $0.23 \pm 0.58$ & $0.97 \pm 0.30$ & $-0.19 \pm 0.30$ & $-1.87 \pm 0.65$ \\
\hline$\hat{y}$ & $-1.65 \pm 0.58$ & $1.88 \pm 0.44$ & $-2.32 \pm 0.91$ & $-0.37 \pm 0.56$ & $2.31 \pm 0.50$ & $0.42 \pm 0.48$ \\
\hline
\end{tabular}


window, within one or two optical cycles at the peak of the driving laser pulse $(38,39)$. For the same reason, we believe that the large deviation from circularity in the cutoff region (Fig. 4C) can be attributed to the unequal intensity contributions from the linear EUV bursts and the breaking of the threefold symmetry, due to the narrow temporal emission window and slight ellipticity in the driving fields.
In summary, we completely characterize the temporal, spectral, and polarization states of circularly polarized harmonics generated by counter-rotating bichromatic driving fields. Using this novel generation scheme, we demonstrate that the circular harmonics consist of a superposition of three linear EUV bursts with alternating polarization directions, repeating each optical cycle, which validates theory in the

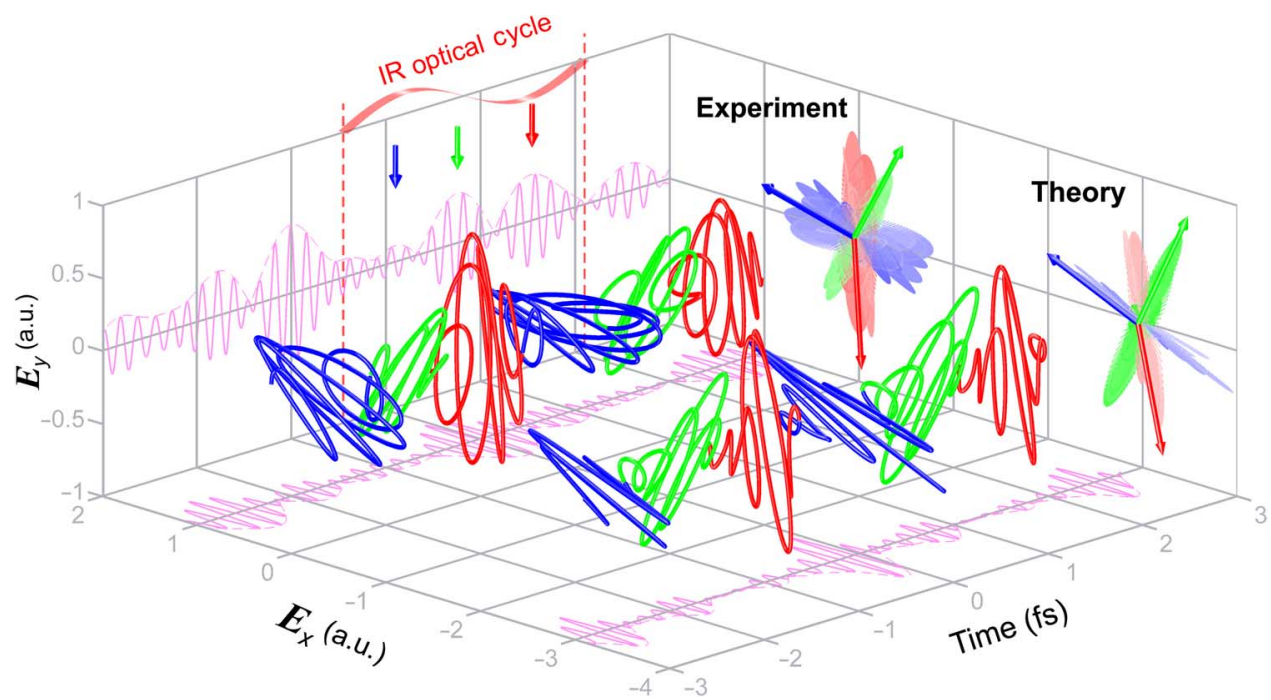

Fig. 3. Experimental reconstruction of the circular HHG pulse train. Three-dimensional field plot of the experimentally reconstructed pulse train of circular HHG, in direct comparison with our numerical simulation. The pulse train from theory is offset by -3 units along the $E_{x}$ axis for illustration. Different colors (red, blue, and green) highlight the three linear bursts with different field orientations within each fundamental IR optical cycle (2.6 fs). The structure of three bursts and an IR optical cycle are also labeled on the $\hat{y}$ projection of the electric field. The carrier field of the experimental pulse train is reconstructed by assuming the phase of the $\hat{x}$ component of the 13th harmonic to be zero.

A

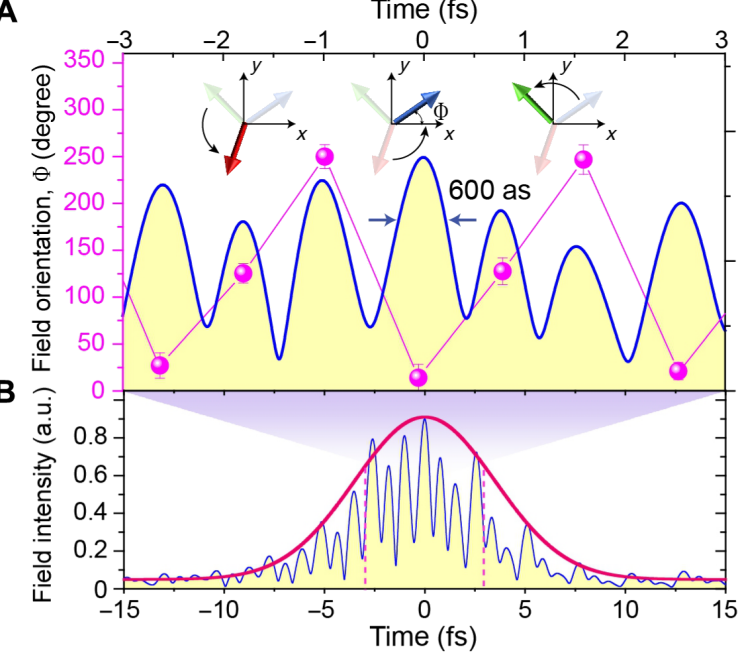

C

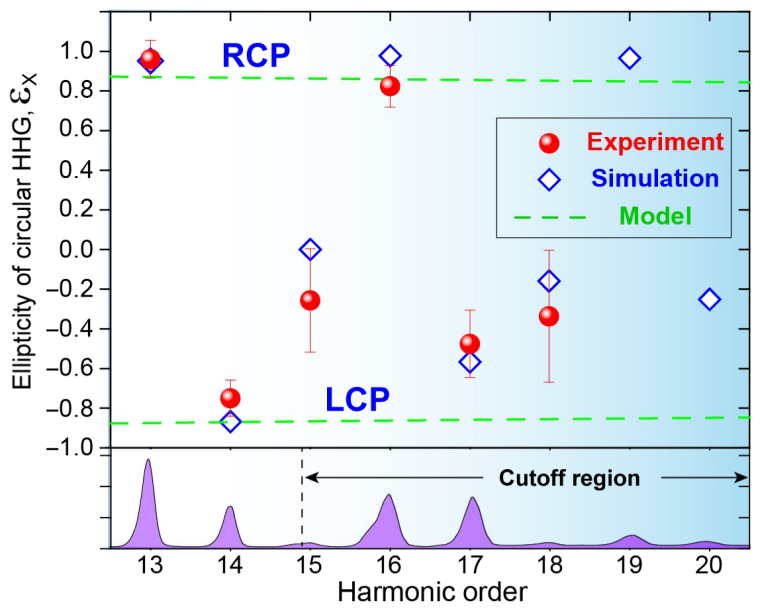

Fig. 4. Field orientation and ellipticity of circular HHG from Ar. (A) Field orientation and intensity profile of the experimentally reconstructed circular HHG pulse train. The orientation of each EUV burst, $\Phi$, is defined as the angle of the electric field relative to the $\hat{x}$ axis, as shown in the inset of (A). $\Phi$ is extracted by averaging the field orientation throughout each burst (see the Supplementary Materials). (B) Overall field intensity profile of the pulse train between -15 and $15 \mathrm{fs}$. The three-burst structure per IR optical cycle ( $2.6 \mathrm{fs})$ can be distinguished throughout the entire pulse train. The red curve represents the envelope of the pulse train with a full width at half maximum of $\sim 8 \mathrm{fs}$. A zoom-in view between -3 and $3 \mathrm{fs}$ is presented in (A). (C) Experimentally measured and theoretically calculated ellipticity of circular HHG from $\mathrm{Ar}$ (see the Supplementary Materials for the formula). The open blue diamonds are the simulation results with $\varepsilon_{1}=1.0$ and $\varepsilon_{2}=0.92$, where $\varepsilon_{1}$ and $\varepsilon_{2}$ are the ellipticities of 780- and 390-nm driving fields, respectively. The green dashed lines represent the results considering spin angular momentum conservation under a perturbative model by Fleischer et al. (13) and Pisanty et al. (37) (see the Supplementary Materials). The HHG spectrum obtained from the experiment is plotted in the lower panel. 
time domain (17). We note that, although some ellipticity has been intentionally introduced in the driving laser field to induce weak $3 m$ th-order harmonics, which is necessary to bridge neighboring harmonic pairs in our current phase retrieval algorithm, this limitation can be removed by implementing a more complicated algorithm such as FROG (frequency-resolved optical gating)-CRAB (26). With this implementation, our approach can be applied to fully characterize the properties of both attosecond pulse trains and broadband isolated attosecond pulses from arbitrary HHG fields and is essential for investigations of coherent attosecond chiral and magnetic dynamics (40), where knowledge of the full temporal structure of the EUV field is important.

\section{MATERIALS AND METHODS}

\section{Optical setup}

The circularly polarized high-order harmonics are generated by propagating an IR beam and its second harmonic into a hollow waveguide filled with Ar gas. The fundamental IR pulses $(780 \mathrm{~nm})$ are derived from a multipass Ti/Sapphire laser system (Dragon, KMLabs), with pulse energy of $2.4 \mathrm{~mJ}$ at a repetition rate of $4 \mathrm{kHz}$. The second harmonic beam with pulse energy of $0.7 \mathrm{~mJ}$ is produced by passing the fundamental laser through a $200-\mu \mathrm{m}$-thick $\beta$-phase barium borate crystal, which then copropagates with the residual IR pulse $(1.2 \mathrm{~mJ})$. Both laser beams are focused into a 1-cm-long capillary waveguide with $150-\mu \mathrm{m}$ inner diameter filled by Ar gas at a pressure of 30 torr. The intensity of the dressing laser field is estimated to be $2.8 \times 10^{11} \mathrm{~W} / \mathrm{cm}^{2}$.

\section{Sample preparation}

An atomically clean $\mathrm{Cu}(111)$ surface is obtained by repeatedly sputtering the sample surface at room temperature with argon ions of $0.7 \mathrm{keV}$ at an incidence angle of $\pm 60^{\circ}$, followed by annealing to $820 \mathrm{~K}$ for $10 \mathrm{~min}$. The sample preparation and measurements are all done in an ultrahigh vacuum chamber with a base pressure of $<5 \times 10^{-10}$ torr. The sample surface quality is confirmed by monitoring the low-energy electron diffraction patterns and the Shockley surface state in the photoemission spectra.

\section{Data analysis}

The original photoemission spectra were first masked to eliminate the contribution of $s p$ band and surface states from the $\mathrm{Cu}(111)$ surface, leaving us with the dominant contributions from the $d$ band with a nearly constant photoemission cross section within the energy range of our experiments (34). We integrate the spectra over the entire measured momentum region and plot the energy-distribution curve as a function of the temporal delay between the circular harmonics and the dressing IR field, which gives us the interferogram as shown in Fig. 2A. The interferogram phase is retrieved by cross-correlation Fourier analysis (41) (see the Supplementary Materials for details).

\section{Phase retrieval}

The derivation of Eq. 1 follows the strong-field approximation (SFA) in recent research $(26,27,33,42,43)$. To retrieve the phases of circularly polarized harmonics, we used a genetic algorithm (44) to search for the correct harmonic phases by minimizing the difference between the calculated and measured interferogram phases. In the genetic algorithm, reproduction is carried out using roulette wheel selection.
Cloning, mutation, and crossover operations are used to increase the diversity of population and to prevent stagnation.

\section{Numerical simulation of circular harmonic generation}

Circular harmonic generation is computed in the dipole approximation using the SFA method, without resorting to the saddle point approximation (35). Coulomb corrections are introduced using the corresponding prefactor (45). The argon wave functions are described as superpositions of Slater-type orbitals resulting from the Roothaan-Hartree-Fock method (46). We take into account the electrons in the three valence orbitals, adding their contributions to the HHG spectrum coherently.

The driving field is described as a superposition of two counterrotating elliptically polarized laser pulses in the form of

$$
E(t)=\sum_{i=1,2} \frac{A_{i}(t) E_{i}}{\sqrt{1+\varepsilon_{i}^{2}}}\left[\cos \left(\omega_{i} t\right) \hat{x}+\varepsilon_{i} \sin \left(\omega_{i} t\right) \hat{y}\right]
$$

where $\varepsilon_{i}$ is the driving laser ellipticity. The driver frequencies correspond to laser wavelengths of $\lambda_{1}=0.78 \mu \mathrm{m}$ and $\lambda_{2}=0.39 \mu \mathrm{m}$, respectively. The temporal envelope $A_{i}(t)$ has a trapezoidal shape with three cycles of linear turn-on, six cycles of constant amplitude (16 fs), and three cycles of linear turnoff (in terms of cycles of $\lambda_{1}$ ). The electric field amplitudes $\left(E_{i}\right)$ have associated intensities of $I_{1}=7.6 \times 10^{13} \mathrm{~W} / \mathrm{cm}^{2}$ and $I_{2}=3.8 \times 10^{13} \mathrm{~W} / \mathrm{cm}^{2}$, which preserve the ratio between two driving fields ( 2:1) used in our experiments. The field intensities are adjusted to match the cutoff energy observed in the experiments.

\section{Statistical analysis}

In the experiments, we measure the interferogram by repeating the pump-probe delay cycle more than 50 times, which gives a consistent interferogram phase spectrum with an SD of $\sim 0.02 \mathrm{rad}$. The errors in Fig. 4 and Table 1 are mainly contributed by the generic algorithm in the phase retrieval process. The robustness of the phase retrieval algorithm is confirmed by performing the retrieval process more than 50 times to guarantee a consistent output for the phase spectra of circular harmonics. The errors of harmonic phases and the corresponding ellipticities are dominantly contributed by the SD in the results of phase retrieval. To properly account for these errors of harmonic phases in the reconstructed pulse train, the reconstruction is repeated 100 times and a random number generator is applied to vary the harmonic phases within the error bar range, which results in a consistent pulse train structure as shown in Fig. 3, with some variation in the field orientation and intensity. The deviation of the field orientation is indicated in the error bars for Fig. 4A.

\section{SUPPLEMENTARY MATERIALS}

Supplementary material for this article is available at http://advances.sciencemag.org/cgi/ content/full/2/2/e1501333/DC1

S1. Experimental setup.

S2. Sample preparation.

S3. Static ARPES spectra from circularly polarized harmonics.

S4. Extraction of the interferogram phases using Fourier analysis.

S5. Phase retrieval using the PROOF algorithm.

S6. Numerical simulation of circular harmonic generation.

S7. Rotation of the driving field to extract the orthogonal components.

S8. Ellipticity of circularly polarized HHG

Fig. S1. Experimental setup.

Fig. S2. Photoelectron spectrum of $\mathrm{Cu}(111)$. 
Fig. S3. Reproducing the phases of the photoelectron interferogram using retrieved HHG phases.

Fig. S4. Photoelectron yields depend on the HHG polarization.

Fig. S5. Normalized 3mth-order intensity compared with numerical simulation.

Fig. S6. Reconstruction of circularly polarized harmonics in comparison with the numerical simulation.

Fig. S7. Rotation of the "clover leaf" driving field.

Fig. S8. Simulation of the EUV field rotation.

\section{REFERENCES AND NOTES}

1. L. Miaja-Avila, G. Saathoff, S. Mathias, J. Yin, C. La-o-vorakiat, M. Bauer, M. Aeschlimann, M. M. Murnane, H. C. Kapteyn, Direct measurement of core-level relaxation dynamics on a surface-adsorbate system. Phys. Rev. Lett. 101, 046101 (2008).

2. K. Klünder, J. M. Dahlström, M. Gisselbrecht, T. Fordell, M. Swoboda, D. Guénot, P. Johnsson, J. Caillat, J. Mauritsson, A. Maquet, R. Taïeb, A. L'Huillier, Probing single-photon ionization on the attosecond time scale. Phys. Rev. Lett. 106, 143002 (2011).

3. X. Zhou, P. Ranitovic, C. W. Hogle, J. H. D. Eland, H. C. Kapteyn, M. M. Murnane, Probing and controlling non-Born-Oppenheimer dynamics in highly excited molecular ions. Nat. Phys. 8, 232-237 (2012)

4. S. Eich, A. Stange, A. V. Carr, J. Urbancic, T. Popmintchev, M. Wiesenmayer, K. Jansen, A. Ruffing, S. Jakobs, T. Rohwer, S. Hellmann, C. Chen, P. Matyba, L. Kipp, K. Rossnagel, M. Bauer, M. M. Murnane, H. C. Kapteyn, S. Mathias, M. Aeschlimann, Time- and angle-resolved photoemission spectroscopy with optimized high-harmonic pulses using frequencydoubled Ti:Sapphire lasers. J. Electron Spectrosc. 195, 231-236 (2014).

5. A. L. Cavalieri, N. Müller, Th. Uphues, V. S. Yakovlev, A. Baltuška, B. Horvath, B. Schmidt L. Blümel, R. Holzwarth, S. Hendel, M. Drescher, U. Kleineberg, P. M. Echenique, R. Kienberger, F. Krausz, U. Heinzmann, Attosecond spectroscopy in condensed matter. Nature 449, 1029-1032 (2007).

6. P. B. Corkum, Plasma perspective on strong field multiphoton ionization. Phys. Rev. Lett. 71, 1994-1997 (1993).

7. K. J. Schafer, B. Yang, L. F. Dimauro, K. C. Kulander, Above threshold ionization beyond the high harmonic cutoff. Phys. Rev. Lett. 70, 1599-1602 (1993).

8. A. Rundquist, C. G. Durfee III, Z. Chang, C. Herne, S. Backus, M. M. Murnane, H. C. Kapteyn, Phase-matched generation of coherent soft X-rays. Science 280, 1412-1415 (1998).

9. C. Boeglin, E. Beaurepaire, V. Halté, V. López-Flores, C. Stamm, N. Pontius, H. A. Dürr, J.-Y. Bigot, Distinguishing the ultrafast dynamics of spin and orbital moments in solids. Nature $\mathbf{4 6 5}$, 458-461 (2010).

10. T. Muraoka, K. Kinbara, T. Aida, Mechanical twisting of a guest by a photoresponsive host. Nature 440, 512-515 (2006).

11. K. S. Budil, P. Salières, A. L'Huillier, T. Ditmire, M. D. Perry, Influence of ellipticity on harmonic generation. Phys. Rev. A 48, R3437-R3440 (1993).

12. V. V. Strelkov, A. A. Gonoskov, I. A. Gonoskov, M. Yu. Ryabikin, Origin for ellipticity of highorder harmonics generated in atomic gases and the sublaser-cycle evolution of harmonic polarization. Phys. Rev. Lett. 107, 043902 (2011).

13. A. Fleischer, O. Kfir, T. Diskin, P. Sidorenko, O. Cohen, Spin angular momentum and tunable polarization in high-harmonic generation. Nat. Photonics 8, 543-549 (2014).

14. O. Kfir, P. Grychtol, E. Turgut, R. Knut, D. Zusin, D. Popmintchev, T. Popmintchev, H. Nembach, J. M. Shaw, A. Fleischer, H. Kapteyn, M. Murnane, O. Cohen, Generation of bright phasematched circularly-polarized extreme ultraviolet high harmonics. Nat. Photonics 9, 99-105 (2015).

15. T. Fan, P. Grychtol, R. Knut, C. Hernández-Garcia, D. D. Hickstein, D. Zusin, C. Gentry, F. J. Dollar C. A. Mancuso, C. W. Hogle, O. Kfir, D. Legut, K. Carva, J. L. Ellis, K. M. Dorney, C. Chen, O. G. Shpyrko, E. E. Fullerton, O. Cohen, P. M. Oppeneer, D. B. Milošević, A. Becker, A. A. Jaroń-Becker, T. Popmintchev, M. M. Murnane, H. C. Kapteyn, Bright circularly polarized soft X-ray high harmonics for X-ray magnetic circular dichroism. Proc. Natl. Acad. Sci. U.S.A. 112, 14206-14211 (2015).

16. S. Long, W. Becker, J. K. Mclver, Model calculations of polarization-dependent two-color high-harmonic generation. Phys. Rev. A 52, 2262-2278 (1995).

17. D. B. Milošević, W. Becker, R. Kopold, Generation of circularly polarized high-order harmonics by two-color coplanar field mixing. Phys. Rev. A 61, 063403 (2000).

18. H. Eichmann, A. Egbert, S. Nolte, C. Momma, B. Wellegehausen, W. Becker, S. Long, J. K. Mclver, Polarization-dependent high-order two-color mixing. Phys. Rev. A 51, R3414-R3417 (1995).

19. D. B. Milošević, W. Becker, Attosecond pulse trains with unusual nonlinear polarization. Phys. Rev. A 62, 011403 (2000).

20. T. Zuo, A. D. Bandrauk, High-order harmonic generation in intense laser and magnetic fields. J. Nonlinear Opt. Phys. 04, 533-546 (1995).

21. A. D. Bandrauk, H. Lu, Controlling harmonic generation in molecules with intense laser and static magnetic fields: Orientation effects. Phys. Rev. A 68, 043408 (2003).
22. C. A. Mancuso, D. D. Hickstein, P. Grychtol, R. Knut, O. Kfir, X.-M. Tong, F. Dollar, D. Zusin, M. Gopalakrishnan, C. Gentry, E. Turgut, J. L. Ellis, M.-C. Chen, A. Fleischer, O. Cohen, H. C. Kapteyn, M. M. Murnane, Strong-field ionization with two-color circularly polarized laser fields. Phys. Rev. A 91, 031402(R) (2015)

23. P. Tzallas, D. Charalambidis, N. A. Papadogiannis, K. Witte, G. D. Tsakiris, Direct observation of attosecond light bunching. Nature 426, 267-271 (2003).

24. P. M. Paul, E. S. Toma, P. Breger, G. Mullot, F. Augé, Ph. Balcou, H. G. Muller, P. Agostini, Observation of a train of attosecond pulses from high harmonic generation. Science 292, 1689-1692 (2001).

25. A. Wirth, M. Th. Hassan, I. Grguraš, J. Gagnon, A. Moulet, T. T. Luu, S. Pabst, R. Santra, Z. A. Alahmed, A. M. Azzeer, V. S. Yakovlev, V. Pervak, F. Krausz, E. Goulielmakis, Synthesized light transients. Science 334, 195-200 (2011).

26. Y. Mairesse, F. Quéré, Frequency-resolved optical gating for complete reconstruction of attosecond bursts. Phys. Rev. A 71, 011401 (R) (2005).

27. M. Chini, S. Gilbertson, S. D. Khan, Z. Chang, Characterizing ultrabroadband attosecond lasers. Opt. Express 18, 13006-13016 (2010).

28. M. Hentschel, R. Kienberger, Ch. Spielmann, G. A. Reider, N. Milosevic, T. Brabec, P. Corkum, U. Heinzmann, M. Drescher, F. Krausz, Attosecond metrology. Nature 414, 509-513 (2001).

29. L. Medišauskas, J. Wragg, H. van der Hart, M. Y. Ivanov, Generating isolated elliptically polarized attosecond pulses using bichromatic counterrotating circularly polarized laser fields. Phys. Rev. Lett. 115, 153001 (2015).

30. D. D. Hickstein, F. J. Dollar, P. Grychtol, J. L. Ellis, R. Knut, C. Hernández-García, D. Zusin, C. Gentry, J. M. Shaw, T. Fan, K. M. Dorney, A. Becker, A. Jaroń-Becker, H. C. Kapteyn M. M. Murnane, C. G. Durfee, Non-collinear generation of angularly isolated circularly polarized high harmonics. Nat. Photonics 9, 743-750 (2015).

31. D. B. Milošević, Generation of elliptically polarized attosecond pulse trains. Opt. Lett. 40, 2381-2384 (2015).

32. D. B. Milošević, Circularly polarized high harmonics generated by a bicircular field from inert atomic gases in the $p$ state: A tool for exploring chirality-sensitive processes. Phys. Rev. A 92, 043827 (2015).

33. T. Ruchon, A. Camper, Notes on attosecond pulse profile measurements with the RABBIT technique. Uvx 2012, 01014 (2013).

34. J. A. Knapp, F.J. Himpsel, D. E. Eastman, Experimental energy band dispersions and lifetimes for valence and conduction bands of copper using angle-resolved photoemission. Phys. Rev. B 19, 4952-4964 (1979).

35. J. A. Pérez-Hernández, L. Plaja, Quantum description of the high-order harmonic generation in multiphoton and tunneling regimes. Phys. Rev. A 76, 023829 (2007).

36. D. B. Milošević, High-order harmonic generation by a bichromatic elliptically polarized field: Conservation of angular momentum. J. Phys. B: At. Mol. Opt. Phys. 48, 171001 (2015).

37. E. Pisanty, S. Sukiasyan, M. Ivanov, Spin conservation in high-order-harmonic generation using bicircular fields. Phys. Rev. A 90, 043829 (2014).

38. K. J. Schafer, K. C. Kulander, High harmonic generation from ultrafast pump lasers. Phys. Rev. Lett. 78, 638-641 (1997).

39. I. P. Christov, M. M. Murnane, H. C. Kapteyn, High-harmonic generation of attosecond pulses in the "single-cycle" regime. Phys. Rev. Lett. 78, 1251-1254 (1997).

40. F. Kelkensberg, W. Siu, J. F. Pérez-Torres, F. Morales, G. Gademann, A. Rouzée, P. Johnsson, M. Lucchini, F. Calegari, J. L. Sanz-Vicario, F. Martín, M. J. J. Vrakking, Attosecond control in photoionization of hydrogen molecules. Phys. Rev. Lett. 107, 043002 (2011).

41. M. Rhudy, B. Bucci, J. Vipperman, J. Allanach, B. Abraham, Microphone array analysis methods using cross-correlations. in ASME 2009 International Mechincal Engineering Congress and Exposition, Lake Buena Vista, FL, 13 to 19 November 2009 (American Society of Mechanical Engineers, New York, 2009), pp. 281-288.

42. C.-H. Zhang, U. Thumm, Attosecond photoelectron spectroscopy of metal surfaces. Phys. Rev. Lett. 102, 123601 (2009)

43. L. B. Madsen, Strong-field approximation in laser-assisted dynamics. Am. J. Phys. 73, 57-62 (2005).

44. D. Whitley, A genetic algorithm tutorial. Stat. Comput. 4, 65-85 (1994)

45. V. P. Krainov, lonization rates and energy and angular distributions at the barriersuppression ionization of complex atoms and atomic ions. J. Opt. Soc. Am. B. 14, 425-431 (1997).

46. E. Clementi, C. Roetti, Roothaan-Hartree-Fock atomic wavefunctions: Basis functions and their coefficients for ground and certain excited states of neutral and ionized atoms, $Z \leq 54$. Atom Data Nucl. Data 14, 177-478 (1974).

Acknowledgments: We thank the staff of the JILA machine shop for their important technical support. Funding: This work was done at JILA. We gratefully acknowledge support from the NSF through the Physics Frontiers Centers Program with grant no. PHY1125844 and the Gordon and Betty Moore Foundation EPiQS (Emergent Phenomena in Quantum Systems) Initiative through Grant GBMF4538 to M.M. C.H.-G. acknowledges support from the Marie Curie International Outgoing Fellowship within the European Union Seventh Framework Programme for Research and Technological Development (2007-2013), under Research Executive 
Agency grant agreement no. 328334. R.K. acknowledges the Swedish Research Council (VR) for financial support. A.J.-B. was supported by grants from the U.S. NSF (grant nos. PHY-1125844 and PHY-1068706). C.H.-G. and L.P. acknowledge support from Junta de Castilla y León (project SA116U13) and MINECO (Ministerio de Economía y Competitividad) (FIS2013-44174-P and FIS2015-71933-REDT). This work used the Janus supercomputer, which is supported by the U.S. NSF (grant no. CNS-0821794) and the University of Colorado, Boulder. P.G. acknowledges support from the Deutsche Forschungsgemeinschaft (no. GR 4234/1-1). Author contributions: M.M. and H.K. conceived and supervised the project. C.C., Z.T., P.M., A.C., R.K., D.Z., and P.G. constructed the experimental setup and conducted the experiment. C.C., Z.T., and O.K. analyzed the data and modeled the phase retrieval algorithm with input from other authors. C.H.-G. carried out the numerical simulation for circular harmonic generation with supervision from L.P. A.B., and A.J.-B. C.C. and Z.T. with M.M. wrote the manuscript with input from all other authors. Competing interests: H.K. and M.M. have a financial interest in a laser company, KMLabs. Data and materials availability: All data needed to evaluate the conclusions in the paper are present in the paper and/or the Supplementary Materials. Additional data may be requested from the authors.

Submitted 25 September 2015

Accepted 2 December 2015

Published 19 February 2016

10.1126/sciadv. 1501333

Citation: C. Chen, Z. Tao, C. Hernández-García, P. Matyba, A. Carr, R. Knut, O. Kfir, D. Zusin, C. Gentry, P. Grychtol, O. Cohen, L. Plaja, A. Becker, A. Jaron-Becker, H. Kapteyn, M. Murnane, Tomographic reconstruction of circularly polarized high-harmonic fields: $3 D$ attosecond metrology. Sci. Adv. 2, e1501333 (2016) 


\section{ScienceAdvances}

\section{Tomographic reconstruction of circularly polarized high-harmonic fields: 3D attosecond}

metrology

Cong Chen, Zhensheng Tao, Carlos Hernández-García, Piotr Matyba, Adra Carr, Ronny Knut, Ofer Kfir, Dimitry Zusin, Christian Gentry, Patrik Grychtol, Oren Cohen, Luis Plaja, Andreas Becker, Agnieszka Jaron-Becker, Henry Kapteyn and Margaret Murnane

Sci Adv 2 (2), e1501333.

DOI: 10.1126/sciadv.1501333

ARTICLE TOOLS

SUPPLEMENTARY MATERIALS

REFERENCES

PERMISSIONS http://advances.sciencemag.org/content/2/2/e1501333

http://advances.sciencemag.org/content/suppl/2016/02/16/2.2.e1501333.DC1

This article cites 44 articles, 4 of which you can access for free http://advances.sciencemag.org/content/2/2/e1501333\#BIBL

http://www.sciencemag.org/help/reprints-and-permissions 